\title{
Thoughts on Tachyon Cosmology
}

\author{
G.W. Gibbons \\ D.A.M.T.P., \\ Cambridge University, \\ Wilberforce Road, \\ Cambridge CB3 0WA, \\ U.K.
}

November 2, 2018

\begin{abstract}
After a pedagogical review of elementary cosmology, I go on to discuss some obstacles to obtaining inflationary or accelerating universes in $\mathrm{M} /$ String Theory. In particular, I give an account of an old "No-Go Theorem" to this effect. I then describe some recent ideas about the possible rôle of the tachyon in cosmology. I stress that there are many objections to a naive inflationary model based on the tachyon, but there remains the possiblity that the tachyon was important in a possible pre-inflationary "Open-String Era " preceeding our present "Closed String Era".
\end{abstract}




\section{Introduction}

The original aim of my lecture was to give a brief outline some recent work and its justification on the possible role of the tachyon in cosmology [23. I also wanted to describe some recent work with Hashimoto and Yi [24], extending some older work with Hori and Yi 25] on the effective-field-theoretic details of the process of tachyon condensation. In particular I intended to describe what we have called the Carollian confinement mechanism for open string states. Since this entails familiarity with the Carroll group I planned to include some some new and old material on that as well.

However I was also asked to cover some more elementary topics in cosmology by way of an introduction. This I have done, but it has lead to a slightly lengthy write-up. Since the subject of cosmology is not short of excellent books and reviews, I have approached the subject from a deliberately idiosyncratic fashion which I hope may appeal to those whose appetite has become somewhat jaded while not misleading the beginner. The reference list, especially when it comes to elementary cosmology, is rather selective, not through any desire to slight the many valuable contributions to the subject but to limit what would be a very large list to a size which I could handle.

\section{Elementary Cosmology}

Before getting on with the main business, I will, as requested by the organisers and various participants, begin with a summary of elementary cosmology. As a first step, we assume the Cosmological Principle which states that the universe is spatially homogeneous and isotropic. Observational support for this assumption comes both from the high degree of isotropy of the cosmic microwave background radiation $(\mathrm{CMB})$, radio observations and the large scale distribution of galaxies.

\subsection{Kinematics of Homogeneity and Isotropy}

Mathematically we reformulate the assumption as stating that locally the metric admits a six-dimensional isometry group acting on three-dimensional spacelike orbits $G / S O(3)$, with $G=S O(4), E(3)$ or $S O(3,1)$. These possibilities are indexed by a quantity $k$ taking the values $1,0,-1$, respectively. Locally, the orbits, i.e. the spatial cross sections, inherit the geometry of $S^{3}, \mathbb{E}^{3}$ or $H^{3}$ respectively with their standard metrics $d s_{k}^{2}$. The metric thus takes the Friedman-LemaitreRoberson-Walker (FLRW) form

$$
-d t^{2}+a^{2}(t) d s_{k}^{2},
$$

where the function $a(t)$ is called the scale factor.

Globally it is always possible to make identifications on the orbits under the action of a discrete sub-group $\Gamma \subset G$, even to the extent that the orbits, $\Gamma G / S O(3)$, in the case $k=0,1$ become compact. Therefore one should avoid labelling the cases $k=1$ "closed" and $k=0,-1$ "open". Future observations 
using the MAP satellite will tell us the extent to which these possibilities are actually realised 20]. In general such identifications destroy isotropy and also homogeneity. The latter may be avoided by taking $k=1$ and identifying $S^{3}$ with $S U(2)$ and taking $\Gamma$ to act exclusively on the left or on the right. One may retain both isotropy and homogeneity by taking $\Gamma$ to be the antipodal map. The spatial sections are then $\mathbb{R P}^{3} \equiv S O(3) \equiv S U(2) / \mathbb{Z}_{2}$. This is sometimes called the elliptic case and was first suggested in the context of relativistic cosmology by Felix-Klein. In fact in 1900, long before the advent of General Relativity, the observational consequences of the universe being spatially $S^{3}$ or $\mathbb{R} \mathbb{P}^{3}$ had been investigated by Karl Schwarzschild (see [20 for an English translation of his paper).

In a general FLRW spacetime the Lorentz group is spontaneously broken down to it's orthogonal subgroup. The cosmological background acts in this respect as a kind of æther. This "æther" is translation invariant, but if $k \neq 0$, the translations do not commute.

For particular scale factors however the metric admits enhanced symmetry: there are additional isometries rendering it homogeneous both in space and time and in fact locally maximally symmetric and thus of constant curvature

$$
R_{\mu \nu \alpha \beta}=\frac{\Lambda}{3}\left(g_{\mu \alpha} g_{\nu \beta}-g_{\mu \beta} g_{\nu \alpha}\right) .
$$

This implies that the metric is also Einstein

$$
R_{\mu \nu}=\frac{\Lambda}{3} g_{\mu \nu} .
$$

The cases are

- de-Sitter spacetime $d S_{4} \equiv S O(4,1) / S O(3,1)$ which happens if $k=1$ and $a(t)=\cosh (H t), k=0$ and $a(t)=\exp (H t)$, and $k=-1$ and $a(t)=$ $\sinh (H t)$, with $H^{2}=\frac{\Lambda}{3}$

- Minkowski spacetime $\mathbb{E}^{3,1}=E(3,1) / S O(3,1)$, which happens if $k=0$ and $a(t)=$ constant or $k=-1$ and $a(t)=t$.

- Anti-de-Sitter spacetime $A d S_{4} \equiv S O(3,2) / S O(3,1)$, which happens if $k=$ -1 and $a(t)=\sin (H t)$ with $H^{2}=-\frac{\Lambda}{3}$.

Some of coordinate systems are purely local. For example the Milne universe (i.e. the $k=-1$ form of Minkowski spacetime) covers only the interior of the future light cone of a point. The light cone corresponds to the coordinate singularity at $t=0$. This model was originally introduced to describe a single creation event for the universe avoiding the use of general relativity. The Steady State universe was invented to achieve the opposite goal. However it (i.e. the $k=0$ form of de-Sitter spacetime) is geodesically incomplete in the past and covers only half of de-Sitter spacetime. There is a past horizon at the coordinate singularity at $t=-\infty$. Thus it is really not past eternal as the founders of 
that theory had supposed and indeed there are now grounds for doubting that inflation can ever be past eternal [19].

In connection with eternal inflation, one might ask what 4-dimensional Lorentzian Einstein spaces can be homogeneous, that is admit a transitively acting isometry group. This requirement is a weak version of what Bondi calls the Perfect Cosmological Principle. The list is not large and moreover contains a surprise. In addition to the previously noted maximally symmetric cases, Minkowski spacetime, $\mathbb{E}^{3,1}$, De-Sitter spacetime $d S_{4}$ and Anti-de-Sitter spacetime $A d S_{4}$, there are the two expected two products $d S_{2} \times S^{2}$ and $A d S_{2} \times H^{2}$. If the cosmological constant is positive, $\Lambda>0$ this is all there are. If $\Lambda \leq 0$ there are two more cases. If $\Lambda=0$ there are homogeneous plane waves, some of which are geodesically complete and another case of Petrov type I. If $\Lambda<0$ one has a Petrov Type N generalized plane wave and another Petrov type III solution.

Thus if eternity is homogeneous and the cosmological constant is positive, then there is really only de-Sitter spacetime or $A d S_{2} \times S^{2}$ to choose from.

Just as the Poincaré group contracts to the Galilei group or the Carroll group 1, 2, the de-Sitter and Anti-de-Sitter groups have interesting contractions which have been classified in 3, 4. One may also obtain the Poincaré group as a contraction of the de-Sitter or Anti-de-Sitter groups and these are in fact the only groups for which this is possible 42 .

\subsection{Kinematics of the Energy Momentum Tensor}

It follows directly from the metric and the Einstein equations or, if one wishes, just from the symmetry assumptions, that the energy momentum tensor takes the form of a perfect fluid

$$
T_{\mu \nu} d x^{\mu} d x^{\nu}=\rho(t) d t^{2}+P(t) g_{i j} d x^{i} d x^{j},
$$

where $\rho(t)$ is called the energy density and $P(t)$ is called the pressure. The Weak Energy Condition requires that the energy density is non-negative

$$
\rho \geq 0 .
$$

Eliminating the coordinate $t$ leads to an equation of state $P=P(\rho)$. Note that this is purely a kinematic statement without necessarily having any ther-

modynamic content. A priori, the pressure need not even be a single valued function of density. If

$$
P=(\gamma-1) \rho,
$$

with $\gamma$ a constant one refers to a polytropic equation of state. More generally one may define the ratio

$$
w=\frac{P}{\rho},
$$

and in the polytropic case $w=(\gamma-1)=$ constant.

The speed of acoustic or sound waves $c_{s}$ is given by Newton's formula

$$
c_{s}^{2}=\frac{\partial P}{\partial \rho} \text {. }
$$


It is real and less than that of light if

$$
1 \leq \gamma \leq 2
$$

If $\gamma=1$, i.e. $P=0$, one speaks of pressure free matter or "dust", $\gamma=\frac{4}{3}$, $P=\frac{1}{3} \rho$, one speaks of "radiation" and if $\gamma=2$, i.e. $P=\rho$ one speaks of "stiff matter". Thus acoustic waves in a radiation fluid travel at a speed $\frac{1}{\sqrt{3}}$. This is the analogue for a gas of photons of "second sound" in a gas of phonons. The acoustic peaks in the BOOMERANG observations of the CMB show clear evidence for the propagation of "second light".

The Dominant Energy Condition allows a negative pressure as well as the usual positive pressure as long as

$$
|P| \leq \rho .
$$

As an example consider a single scalar field $\phi$ with potential $V(\phi)$. If $\phi$ depends only on time then

$$
\begin{aligned}
& \rho=\frac{1}{2} \dot{\phi}^{2}+V(\phi), \\
& P=\frac{1}{2} \dot{\phi}^{2}-V(\phi) .
\end{aligned}
$$

If the kinetic energy dominates we have stiff matter, $P=\rho$, with the maximal possible positive pressure. If the potential energy dominates and we have the greatest possible tension or negative pressure $P=-\rho$.

\subsection{Consequences of the Einstein equations}

The Einstein equations provide three equations.

- Raychaudhuri's equation

$$
\frac{\ddot{a}}{a}=-\frac{4 \pi G}{3}(\rho+3 P)
$$

- Friedman's equation

$$
\frac{\dot{a}^{2}}{a}+\frac{k}{a^{2}}=\frac{8 \pi G}{3} \rho
$$

- The First Law

$$
\dot{\rho}+\frac{3 \dot{a}}{a}(\rho+P)=0 .
$$

The Bianchi identity implies that of these only two are independent. The "First Law" expresses the fact that the energy in a co-moving volume $a^{3} \rho$ is not conserved during the expansion of the universe. If one assumes that the matter is in local thermodynamic equilibrium and passes adiabatically through a succession of equilibrium states whose equilibrium pressure is given exactly by $P$, then one may deduce that the entropy in a co-moving volume is conserved. In practice the matter is never in complete equilibrium, moreover it is 
always tending to the equilibrium configuration of constant entropy at a lower and lower energy, i.e at a lower and lower temperature. This is why naive nineteenth century ideas about the "Heat a Death of the Universe" were essentially misguided. They were predicated on the assumption that the matter in the universe was both static and isolated.

If the equation of state is known one may integrate the first law to obtain the pressure as a function of the scale factor. In the polytropic case one obtains

$$
P \propto \frac{1}{a^{3 \gamma}} .
$$

From this it follows that if the universe is expanding then the density of radiation falls of with one more power of the scale factor $a$ than pressure free matter, which will therefore ultimately dominate.

\section{$2.4 \quad$ Future and Past Horizons}

We now turn to light propagation and horizons. If $r$ is the radial coordinate on $S^{3}, \mathbb{E}^{3}$ or $H^{3}$ with us taken as the origin, then by symmetry, radial null geodesics are given by $r=\eta$ with

$$
d \eta=\frac{d t}{a(t)}
$$

The coordinate $\eta$ is called conformal time because using it, the FLRW metric becomes explicitly conformally static

$$
a^{2}(t)\left(-d \eta^{2}+g_{i j} d x^{i} d x^{j}\right)
$$

In fact the FLRW metric is also conformally flat but we shall not need that fact here.

To read of the causal structure it suffices to consider the Penrose diagram i.e. the orbit space of the $S O(3)$ action, which has coordinates $r \geq 0$ and $\eta$ in which light rays are at 45 degrees. Thus if the polytropic index is a constant so that $\rho \propto \frac{1}{a^{3 \gamma}}$ we define $y=a^{\frac{3 \gamma-2}{2}}$

$$
\frac{d^{2} y}{d \eta^{2}}+k\left(\frac{3 \gamma}{2}-1\right)^{2} y=0
$$

If $k=1$, this is a simple harmonic oscillator with total duration from Big Bang to Big Crunch equal to $\frac{2 \pi}{|3 \gamma-2|}$. Thus for dust, $\gamma=1$, a lightray will just circumnavigate an $S^{3}$ in that time, while for de-Sitter spactime with $\gamma=0$, it would only get half way. The same is true of radiation for which $\gamma=\frac{4}{3}$, while for stiff matter, $\gamma=2$, it only gets one quarter of the way around.

The structure of the conformal boundary is determined by the range of $\eta$. As originally clarified by Rindler,

- If the universe expands forever and :

$$
r_{+}(t)=a(t) \int_{t}^{\infty} \frac{d t^{\prime}}{a\left(t^{\prime}\right)}<\infty
$$


there will be a future event horizon, that is a null hypersuface separating events which the observer at $r=0$ will see from those he or she will never see. In this case the future boundary at $t=\infty$ is spacelike. The standard example of a cosmological spacetime with a future horizon is de-Sitter spacetime. In this case the (observer dependent) horizon is also Killing horizon and its area $A_{+}(t)=4 \pi a^{2}(t) r_{+}(t)$ is constant with time. In general, if the weak energy condition holds, $A_{+}(t)$ is non-decreasing. In the static case one has an associated constant temperature $T_{+}=\frac{2 \pi}{H}$. It is reasonable to assume that if $A_{+}(t)$ does not change too fast one simply replaces $\frac{1}{H}$ in this expression by the instantaneous Hubble radius. One usually ascribes a non-decreasing entropy

$$
S(t)=\frac{1}{4 G} A_{-}(t),
$$

to each co-moving observer's horizon and interprets it as a measure of the total information lost to he or she up to time $t$.

- If the scale factor vanishes at $t=0$, a Big-Bang singularity, and

$$
r_{-}(t)=a(t) \int_{0}^{t} \frac{d t^{\prime}}{a\left(t^{\prime}\right)}<\infty,
$$

then there will be a past horizon, that is a null hypersurface separating events with which an observer at $r=0$ can have been in causal contact since the Big Bang from those he or she cannot have been. In this case there is a singular spacelike past boundary at $t=0$. Events with time coordinate $t$ and radius $r<r_{-}(t)$ are said to be inside the particle hori$z o n$. If identified with the points on co-moving geodesics for which $r$ is independent of time, the entire past geodesic is outside the past light cone of the observer at the origin. Indeed a light ray starting from $t=0$ and $r=0$ just reaches the particle horizon at any subsequent time.

Note that to calculate the particle horizon radius $r_{-}(t)$ at time $t$ requires knowledge of the scale factor at all previous times. In other words it is an antiteleological concept, just as the future horizon is a teleological concept. It is a widespread, misleading and incorrect practice to muddle the particle horizon with the more or less directly observable and instantaneously defined Hubble radius

$$
r_{H}(t)=\frac{a(t)}{\dot{a}(t)} .
$$

If the scale factor goes to zero like $t^{p}$ near $t=0$, then there will be a particle horizon if $p<1$. For a $k=0$ universe with a polytropic equation of state, the Einstein equations imply

$$
p=\frac{2}{3} \text {. }
$$

Regardless of the actual value of $k$, it is almost always a good approximation to set it to zero near a Big Bang because the term $\frac{k}{a^{2}}$ in the Friedman equation (14) is negligible compared with the other terms. 


\subsection{The Horizon Problem}

This gives rise to the Horizon Problem. The point is that the present day observed CMB photons at temperature roughly $3 \mathrm{~K}$ arrive unscattered from the spherical intersection $S_{l s}$ of our past light cone with the Hypersurface of Last Scattering $t=t_{l s}$, red shifting by factor $1+z=\frac{a(o)}{a\left(t_{l s}\right)} \approx 10^{3}$ on the way. Note that they keep their almost perfectly thermal spectrum purely by means of the kinematic effect of their redshift. Before last scattering $t=t_{l s}$ they are presumed to have been in thermal contact. Observations of the CMB reveal that the temperature on $S_{l s}$ was uniform to better than one part in $10^{4}$. This uniformity is a puzzle if one assumes that the universe was radiation dominated all the way back to a Big Bang before $t=t_{l s}$ ( i.e. $p=\frac{1}{2}$ ) because the horizon radius $r_{-}\left(t_{l s}\right)$ calculated under that assumption is about 80 times smaller than the proper diameter $2 a\left(t_{l s}\right) r_{l s}$ of the last scattering sphere $S_{l s}$. In other words, under this assumption, the past light cones of most pairs of points on $S_{l s}$ never intersected.

The recent BOOMERANG and MAXIMA data on the deviations of the temperature of the CMB from isotropy allow us to sharpen this argument. Roughly speaking, such second light behaves like

$$
\frac{\delta T}{T}=\int d^{3} \mathbf{k} e^{i \mathbf{k} \cdot \mathbf{x}}\left[A(\mathbf{k}) \cos \left(\frac{k \eta}{\sqrt{3}}\right)+B(\mathbf{k}) \sin \left(\frac{k \eta}{\sqrt{3}}\right)\right] .
$$

The cosine mode is known to cosmologists as the "iso-curvature" mode and the sine mode as the "adiabatic" mode. The physical distinction between them is that in the latter case the ratio of baryon number to photon number is independent of position whereas in the former it depends upon position. Moreover for "causal" perturbations one expects that the cosine mode is absent because it varies non-trivially at the largest scales, i.e. at the smallest possible values of $k=|\mathbf{k}|$. If one decomposes the temperature $T=T(\theta, \phi)$ on the sphere of last scattering $S_{l s}$ into spherical harmonics

$$
\frac{\delta T}{T}=\sum a_{l m} Y_{l}^{m}(\theta, \phi)
$$

one finds so-called peaks in the amplitudes, the lowest at $l \approx 200$. These can only be explained if the sine mode is absent, thus establishing that the primordial fluctuations have scales much larger than could have arisen from causal processes if the past horizon was roughly the Hubble radius at that time.

A more detailed examination of the data reveals more. The universe is almost flat and the content roughly $30 \%$ cold dark non-baryonic matter, and $70 \%$ matter with negative pressure and $w<-.5$.

\subsection{Inflation}

The theory of Inflation seeks to remedy this, and other defects of the Standard Cosmological Model by postulating a much more rapid growth of the scale factor 
sometime before $t_{l s}$; growth at least as fast as $t$. If true, this means that the scale factor must have accelerated some time before $t_{l s}$,

$$
\ddot{a}>0 .
$$

It follows from the Raychaudhuri equation, that the pressure must have been at least as negative as $-\frac{1}{3} P$. In other words, the universe must have anti-gravitated in the past. Using

$$
\rho+3 P=2\left(T_{00}-\frac{1}{2} g_{00} T_{\lambda}^{\lambda}\right)=\frac{1}{4 \pi G} R_{00},
$$

the Raychaudhuri equation (13) may be written as

$$
\frac{\ddot{a}}{a}=\frac{1}{3} R_{00},
$$

we need

$$
R_{00}<0 .
$$

In the language of energy conditions, we need a massive violation of the Strong Energy Condition in the past.

Observations of distant Type 1A supernovae, which essentially use the curvature of the redshift magnitude or Hubble diagram, assuming that this class of super-novae provide us with standard candles whose light is unscattered on its way to us, also seem to indicate that the universe is accelerating at the present epoch. To explain this anti-gravitating behaviour one is forced to accept directly from Raychaudhuri's equation (13), which is no more than a statement of Newton's law of gravitation, that there must be massive violation of the strong energy condition, with large negative pressures at late times. Indeed if one models the data one finds that about $70 \%$ of the energy density is of this exotic form, the bulk of the remaining matter is dark, non-baryonic and pressure free and less than $4 \%$ is ordinary baryonic matter.

\section{The Inflaton}

The standard way of arranging for a violation of the strong energy condition is to postulate the existence of one or more hitherto unobserved scalar fields admitting everywhere non-negative potentials usually with an absolute minimum for which the potential either vanishes or is very small. Because potentials have dimensions mass to the power 4 , what is required to get a suitable non-vanishing value at late times is a mass scale near the minimum less than $10^{-4} \mathrm{eV}$. Curiously, this is rougly the order of magnitude of some neutrino masses, in fact which perhaps lends greater credence to the idea.

The simplest case is just one scalar field, called in this context an inflaton. It is usually assumed to start near the top of a fairly flat potential and to roll slowly down to the minimum of the potential. The potential needs to be fairly 
flat so that sufficiently many e-folds ( at least 60 ) take place before the minimum is reached.

During slow rolling the second derivative term in the equation of motion

$$
\ddot{\phi}+\frac{3 \dot{a}}{a} \dot{\phi}+\frac{\partial V}{\partial \phi}=0
$$

is neglected so

$$
\frac{3 \dot{a}}{a} \dot{\phi}+\frac{\partial V}{\partial \phi} \approx 0
$$

and the Friedman equation is approximated by

$$
\frac{\dot{a}^{2}}{a^{2}} \approx \frac{8 \pi G}{3} V(\phi) .
$$

Given $V(\phi)$ one may now solve (321) and (33) in what is called the Slow Rolling Approximation to find $a(t)$. I will not give the easily derived formulae here. It is important to realise that because $V(\phi)$ is not constant during slow rolling, the inflation is not exponential and so the de-Sitter metric with $H=\sqrt{\frac{8 \pi G V(\phi)}{3}}$ is only an approximation to the actual metric at that time.

An interesting simple case for which an exact solution is available is given by [27].

$$
V=V_{0} \exp (\alpha \phi)
$$

If $k=0$, one has a solution with

$$
V \propto \frac{1}{t^{2}}, \quad a(t) \propto t .
$$

In string theory, one may think of $\phi$ as the dilaton. The solution is then a timelike version of the usual spacelike linear dilaton vacuum. In string conformal frame, the metric is flat.

\section{1 "Reheating"}

Slow roll over is usually supposed to terminate with a period during which the inflaton executes damped oscillations near the bottom of the potential. During this period its coupling to the matter of the Standard Model of Particle Physics leads to something called in the characteristically confused and muddle and misleading jargon of cosmologists which we encountered when applied to the Hubble radius, "re-heating" since it is not clear, nor more damningly very relevant, whether the universe was ever heated before this time. (Similar strictures apply to the terms "recombination" and "adiabatic").

Polemic aside, the main point is to get some of the energy in the oscillations of the inflaton to produce a radiation dominated universe at a suitably high temperature so that baryon-violating processes can give rise to the puzzling small number of baryons that we both see and love today compared with the very large number of photons in the CMB. 


\subsection{Planck Scale Inflation}

To get a suitable inflationary scenario, not only must one assume that the potential $V(\phi)$ is such as to ensure sufficient inflation, one also needs to ensure that the transition to a radiation era is not too abrupt, otherwise more gravitational waves will be produced than are compatible with the isotropy of the CMB. This is usually taken to bound below the value of the Hubble radius during inflation. If $m_{p}$ is the Planck mass then one needs roughly

$$
\frac{1}{H}>10^{-5} \frac{1}{m_{p}} .
$$

In other words naive models involving Planck scale inflation are very probably ruled out [?].

\section{Machian Considerations, Chronology Protec- tion and the Spontaneous Breakdown of Lorentz- Invariance}

Many cosmologists in the past have worried about "Mach's Principle". This can be taken in many ways. I prefer to think of the "Machian Coincidence". This is the fact that locally, without reference to any astronomical observations, one may define a non-rotating inertial frame, i.e one in which the usual laws of Newtonian mechanics hold, while astronomically we may define a frame with respect to which distant glaxies are at rest and one finds that these two frames coincide. Traditionally the first frame is determined using a version of Newton's bucket experiment. The second may nowadays be determined in terms of the last scattering surface rather than the distant stars or galaxies.

The basic example of a spactime in which these two standards of rest disagree is the well known Goedel solution. It is homogeneous, and hence stationary. This universe, which is spatially non-compact, rotates about every point. However it admits closed timelike curves. A less well-known but in some ways much more striking example was constructed by Ozsvath and Schucking [30. It is also homogeneous but the space sections are squashed $S^{3}$ 's. More importantly, it is causally well behaved. In particular it has no CTC's.

Some years ago, Hawking [13], by considering the transverse Doppler effect, argued that the extent to which the two frames agree is such that the universe can have completed only a very small, fraction of a rotatation since the epoch of combination at which the cosmic plasma became transparent. The original bounds of Hawking have been strengthened somewhat by Barrow, Juskiewicz and Sonada [14.

One mathematical formulation of the Machian coincidence is to consider the average 4 -velocity $\mathbf{U}$ of the matter in the universe. This may be defined as the timelike eigen-direction of the energy momentum tensor. Inertial frames are Fermi-Walker propagated along the integral curves of $\mathbf{U}$. Given the vector 
field $\mathbf{U}$ one may also define its vorticity $\omega=\star u \wedge d u$, where $u$ is the one form obtained from the vector field $\mathbf{U}$ by lowering an index using the metric. The Machian Coincidence is essentially the fact that our present vorticity is very small. One explantion for this fact comes from Inflation as pointed out by Ellis and Olive [15]. Their point is that as the universe expands, then by angular momentum conservation, the vorticity necessarily decreases. Moreover according to inflation, there was probably no initial vorticity to begin with. The first argument holds in almost all inflationary scenarios and the second also holds in inflationary scenarios based on single scalar field. In that case vorticity perturbations necessarilly vanish.

As an aside, let me remark that a striking feature of of many spacetimes with non-vanishing vorticity, which has been known almost since the beginnings of general relativity, is the existence in them of closed timelike curves. Because of the expansion of the universe, violations of Mach's coincidence now require large vorticities at early times. If those large vorticities entailed the existence of CTC's and some mechanism, along the lines of that invoked in Hawking's Chronology Protection Conjecture holds, that might have prevented the occurrence of CTC's thus providing an alternative explanation: the large vorticiy could not have been generated in the first place. For further references and a calculation supporting this idea see [39].

The absence of vorticity implies, a velocity potential, and hence, if the fundamental group of spacetime is trivial, a time function, i.e. a function which increases along every timelike curve. The existence of such a function, which is defined only up to a re-parameterization, guarantees that there are no CTC's. The time-function also provides an absolute rest frame or æther. It may be thought of as the analogue of the Higgs field, responsible for the spontaneous breakdown of Lorentz-invariance, itself somewhat of a Machian conundrum. A time function is also what is needed to resolve the much discussed Problem of Time in quantum cosmology. In inflationary scenarios these functions are performed by the mysterious inflaton. In the theories we are about to describe it is the tachyon field which is responsible. For some recent ramifications of this idea, the reader is directed to [37.

\section{$5 \quad$ M-Theory and Cosmology}

The elementary introduction makes it clear that if we are to make contact between cosmology and fundamental theory, such as M/String Theory we need to violate the strong energy condition and more particularly, obtain scalar fields with positive potentials. This is a notoriously difficult thing to do. It is not just that de-Sitter spacetime is not a supersymmetric background and so reliable quantum calculations like the those around Anti-de-Sitter spacetime cannot be done. The problem is much more basic than that. It is that pure supergravity theories, without additional supermatter in arbitrary dimensions satisfy the strong energy condition and thus do not permit accelerating behaviour. As a consequence they also do not allow oscillatory or cyclic behaviour for the scale 
factor. In fact for compact universes, Tipler has adapted the techniques used to prove the Hawking-Penrose Singularity Theorems to show that the strong energy condition rules out any type of recurrence behaviour [32]. For related reasons, the negative tension branes required in certain brane-world scenarios violate even the weak positive energy condition and cannot come out of supergravity theories 40.

As is well known, if a cosmological term is possible it must be negative, not positive. The reasons for this seem to be rather deep. One explanation comes from thinking of the theories in higher dimensions, specifically 10 and 12 dimensions. This will be the subject of the next section. It is well known that gauged supergravities in 4 spacetime dimension for vector gauging have negative cosmological constant

$$
\Lambda=-\frac{3 e^{2}}{4 \pi G} .
$$

Curiously for axial gauging Freedman [49] showed that one has a positive cosmological constant

$$
\Lambda=-\left(\gamma_{5}\right)^{2} \frac{3 e^{2}}{4 \pi G} .
$$

However such axial gauging leads to anomalies and hence to non-viable theories. It seems that this problem can arise in hybrid inflation models in which FayetIlliopoulos terms come into play.

\section{Compactification and a No-Go Theorem}

In what follows, I shall recall some facts which have been known since the early '80's but which still seem relevant today [35]. Despite the passage of time, compactification is still a very imperfectly understood process from the physical point of view. One speaks of an $N$-dimensional spacetime $M$ being compactified if it is a (possibly warped) product of a Lorentzian spacetime $X$ with a Riemannian spacetime $Y$ :

$$
M=X \times_{W} K .
$$

with a metric given in local coordinates $x^{M}$ which split as $x^{\mu}$ for $X$ and $y^{m}$ for Y

$$
g_{M N} d x^{M} d x^{N}=W^{2}(y) g_{\mu \nu}(x) d x^{\mu} d x^{\nu}+g_{m n}(y) d y^{m} d y^{n},
$$

where $W(y)$ is called the warp factor. This statement gives no insight into the process of compactification itself, which was presumably a dynamical process which occurred in the early universe, possibly as some sort tunnelling process " $e x$ nihilo or as a semi-classical approximation to the wave function of the universe according to the "no boundary" proposal. An alternative picture is obtained if one views the present universe as the outcome of some sort of dynamical 
collapse to a branelike configuration. More generally one might envisage that $M$ contains more than one brane which may also undergo collision. In the former two cases at least it is natural to assume that $Y$ is compact, complete and non-singular without boundary $\partial Y=0$ and that the warp factor $W(y)$ is a smooth and no-where vanishing function on $Y$. In the latter cases, $Y$ may well be non-compact and $W$ might well vanish at the location of Killing horizons.

Rather general restrictions on the form that the metric (40) may take are obtained if one recalls (or checks that) that for all pure supergravity models the bosonic energy momentum tensor $T_{M N}$ satisfies the Strong Energy Condition. The Einstein equations in $M$ read:

$$
R_{M N}=8 \pi G_{N}\left(T_{M N}-\frac{1}{N-2} g_{M N} T_{L}^{L}\right) .
$$

and the strong energy condition is the statement that for all non-spacelike vectors $T^{M}$

$$
R_{M N} T^{M} T^{N} \geq 0
$$

or in any local coordinate system

$$
R_{00} \geq 0 .
$$

Physically the Strong Energy Condition is the condition that locally gravity is attractive. It amounts to saying that any tensions or negative pressures, themselves a rather unusual occurrence, can never exceed $\frac{1}{N-1}$ times the energy density. In other words, a medium not satisfying the Strong Energy Condition must be one supporting extremely large tensions or negative pressures. Usually such a medium would be expected to be highly unstable. It is curious that in pre-relativistic discussions of Newtonian Gravity, a stress tensor was ascribed to the æther with precisely this odd property (see [26]) and it was felt, for example by Clerk Maxwell, to be odd at the time [41.

It is not immediately obvious why this restriction (considered via 41) as a condition on the energy momentum tensor is satisfied for all by all the usual bosonic energy momentum tensors considered in physics, built for example from p-form field strengths, $p \geq 2$, and for minimally coupled scalar fields with the exception of scalar fields with a potential which can somewhere become positive. Moreover the inequality is strict in the sense that if $R_{00}=0$ then the energy momentum tensor must vanish and hence the bosonic fields must actually vanish. For minimally coupled massless scalars without potentials, one finds that they can depend on space but not on time. In checking the Strong Energy Condition in string theory, it is essential to work in Einstein conformal frame, in which the dilaton is minimally coupled. In string conformal frame, second derivatives of the dilaton appear on the right hand side of Einstein's equations and nothing can then be said about the sign of $R_{00}$.

It is important to note that if the Strong Energy Condition is satisfied for the energy momentum tensors then it is also satisfied for arbitrary positive 
linear combinations. Thus for a multi- component system of fields, possibly a statistical mixture, it is necessarily satisfied if the individual components of the mixture satisfy it. For this reason it is satisfied for all the gaseous and liquid media (so-called "perfect fluids") that one typically considers in cosmology. It also implies that the exponential factors involving dilatons, which appear ubiquitously in supergravity lagrangians, do not prevent the Strong Energy Condition holding if it holds for the individual pieces in the Lagrangian.

One should also note that the Strong Energy Condition 8 is independent of the Dominant Energy Condition. This states that in all orthonormal frames $T_{00} \geq\left|T_{M N}\right|$, or more geometrically if $T^{M}$ lies inside or on the future light cone then so does $T^{M}{ }_{N} T^{N}$. The Dominant Energy Condition may be interpreted as requiring that matter may not move superluminally. It again is satisfied by all the usual bosonic energy momentum tensors, including minimally coupled scalars with positive potentials. Evidently it may violated by minimally coupled scalars with negative potentials. The Dominant Energy condition is used to prove the Positive Energy Theorem which in turn implies that the purely gravitational force between isolated systems is always attractive. In other words it rules out the possibility of long range gravitational repulsions. The Strong Energy Condition rules out antigravity in a more local sense in that, as we shall see shortly, it says that the source of the Newtonian potential is always locally of the same sign. Applied to cosmology it implies that the acceleration of the universe is always negative. In particular it is incompatible with de-Sitter or de-Sitter-like behaviour for the spacetime metric $g_{\mu \nu}$ on $X$.

To see this in detail we use the formulae relating the Ricci tensor $R_{M N}$ of $M$ to those, ${ }^{X} R_{\mu \nu}$ and ${ }^{Y} R_{m n}$ of $X$ and $Y$ respectively. They are

$$
R_{\mu \nu}={ }^{X} R_{\mu \nu}+g_{\mu \nu} \frac{1}{p+1} \frac{1}{W^{p+1}}{ }^{Y} \nabla^{2}\left(W^{p+1}\right),
$$

and

$$
R_{m n}={ }^{Y} R_{m n}-\frac{1}{W}{ }^{Y} \nabla_{m}{ }^{Y} \nabla_{n}\left(W^{p+1}\right),
$$

where ${ }^{Y} \nabla_{m}$ is the Levi-Civita covariant derivative on $Y$ and ${ }^{Y} \nabla^{2}$ the Laplacian on $Y$ and the dimension of $X$ is $p+1$. If $p=0$, then we may with no loss of generality put $g_{00}=-1$, the warp factor is essentially the Newtonian potential of the static $N$ dimensional metric on $M$ and (44) becomes the relativistic Poisson equation. If $R_{00} \geq 0$ then it will have a source with the standard sign for a gravitational field. As a consequence the total gravitational mass of such a static spacetime spacetime is non-negative.

Now let's allow $p>0$, and work in an arbitrary orthonormal frame. We get

$$
R_{00}={ }^{X} R_{00}-\frac{1}{p+1}{\frac{1}{W^{p-1}}}^{Y} \nabla^{2}\left(W^{p+1}\right) .
$$

If the "internal " manifold $Y$ is compact and without boundary and $W$ smooth and nowhere vanishing on it, then we may multiply equation (46) by $W^{p+1}$ and integrate over $Y$. The term involving ${ }^{Y} \nabla^{2}$ vanishes and we we deduce that 


$$
{ }^{X} R_{00} \geq 0 .
$$

In other words, the Strong Energy Condition is hereditary. If it holds on $M$ and we compactify on $Y$ then it continues to hold on $X$. This is of course obvious for a strict metric product for which the warp factor $W$ is constant. What we have shown is that it remains true for a non-constant warp factor, as long as $Y$ is compact non-singular and without boundary.

If for example $X$ is a maximally symmetric spacetime it must be an Einstein space with $R_{\mu \nu}=\Lambda g_{\mu \nu}$ and we deduce that necessarily the cosmological constant is non-positive

$$
\Lambda \leq 0
$$

Moreover if we want $X$ to be Minkowski spacetime then we can easily deduce from (46), that since

$$
\int_{Y} W^{p+1} R_{00} \geq 0
$$

in fact $R_{00}=0$ and hence $R_{M N}=0$ and thus the higher dimensional spacetime must be Ricci flat. The bosonic fields must vanish. It is now clear that $W^{p+1}$ is a harmonic function on the closed manifold $Y$ and hence it must be constant. The compactification must in fact be the metric product of Minkowski spacetime with a closed Ricci flat manifold. Moreover it follows that, unless it splits as the (possibly local) metric product of a circle and a lower dimensional Ricci flat manifold $Y^{\prime}$, that the internal manifold $Y$ can admit no continuous isometries. To see why this is true, recall that from Killing's equations, it follows that any Killing vector field $K^{m}$ satisfies

$$
{ }^{Y} \nabla^{2} K^{m}-R^{m}{ }_{n} K^{n}=0 .
$$

Multiplying (50) by $K_{m}$ and integrating over $Y$ shows that $K^{m}$ must be covariantly constant,

$$
{ }^{Y} \nabla_{m} K_{n}=0 .
$$

In particular we find that $g_{m n} K^{m} K^{n}$ is constant and that $K_{m}=\frac{\partial}{\partial y^{m}} \theta$ for some function $\theta$. Using coordinates in which $K^{m}=\delta_{1}^{m}$ we deduce that $y^{1}=\theta$ and the metric may be written as

$$
g_{m n} d y^{m} d y^{n}=d \theta^{2}+g_{m n} d y^{m} d y^{n},
$$

where the summation on the right hand side is from 2 to the dimension of $Y$ and $g_{m n}$ is independent of $\theta$.

We may apply these results to either eleven or ten dimensional supergravity. If we want $X$ to be Minkowski spacetime and in addition we want some 
supersymmetry, then $Y$ must be Ricci flat and admit some covariantly constant spinor fields. If it is irreducible and seven dimensional it must have holonomy $G_{2}$ and if it is six dimensional it must have holonomy in $S U(3)$. Note that one can in fact deduce that if $Y$ is irreducible and that it admits a covariantly constant spinor then it must be Ricci flat. We saw regardless of whether we had Killing spinors, that $Y$ must be Ricci flat and admit no continuous isometries.

Note that in ten-dimensional string theory, as opposed to ten dimensional supergravity, we have extra complications. Yang-Mills fields are present which are not coupled minimally to the metric but in a more subtle way to the curvature involving Chern-Simons terms. The consequence is that if we start with a purely supergravity solution and then identify the Yang-Mills connection with (part of) the Levi-Civita connection, then it will automatically satisfy the YangMills equations of motion. Moreover the source in the dilaton equations of motion vanishes, and it thus is consistent to take the dilaton to be constant, thus evading the No-Go theorem which would otherwise follow. The full curvature modified Einstein equations are now much more complicated and the simple arguments given above do not directly apply. Thus in string theory the YangMills sector combined with the curvature contributions does in effect conspire to violate the Strong Energy Condition. Similar remarks apply to M-theory, as opposed to eleven-dimensional supergravity. If extra curvature terms are introduced then the simple arguments given above need not apply.

Of course a No-Go Theorem is no better than the assumptions that go into it. If one considers non-compact internal spaces [48] or couples super-matter to supergravity [47] then one can obtain de-Sitter space as a solution of the classical equations of motion.

\section{The Tachyon}

In the light of these rather discouraging results, it seems clear that it is worth exploring a new idea. Moreover it is very striking that an intrinsic part of string theory, both open and closed, and apparent from its very beginnings in Regge theory, is the existence of tachyons. It is true that in closed string theory they are projected out, but even there the projection mechanism has only been checked explicitly in perturbation theory to 2 loops! In open string theory tachyons abound. Great progress has been made of late in understanding their significance. I shall not attempt to describe any of that work in detail. I shall merely take away from it the idea that one the existence of a tachyon in the perturbative spectrum indicates that the perturbative vacuum is unstable and that there exist a true vacuum, with zero energy density, toward which a tachyon field $T(x)$ naturally moves. Moreover it seems that aspects of this process can be capture by comparatively simple effective field theory models. Perhaps the simplest is that proposed by Sen. It has (in units in which $2 \pi \alpha^{\prime}=1$ ) the Lagrangian

$$
L=-V(T) \sqrt{1+g^{\mu \nu} \partial_{\mu} T \partial_{\nu} T} \sqrt{-\operatorname{det}\left(g_{\mu \nu}\right)}
$$




$$
=-V(T) \sqrt{-\operatorname{det} G_{\mu \nu}},
$$

where what we shall call the tachyon metric is given by

$$
\begin{aligned}
G_{\mu \nu} & =(G)_{\mu \nu} \\
& =g_{\mu \nu}+\partial_{\mu} T \partial_{\nu} \cdot T
\end{aligned}
$$

The potential $V(T)$ is taken to be non-negative have a unique local maximum at the origin $T=0$ and a unique global minimum away from the origin at which $V$ vanishes. In the most interesting case the global minimum is taken to lie at $|T|=\infty$. Obviously more complicated potentials may be contemplated but this is the simplest case to begin with. Small fluctuations around the "false vacuum" at $T=0$ have negative mass squared and so it is unstable. How does the system evolve?

The equation of motion is

$$
\left(g^{\mu \nu}-\frac{\partial^{\mu} T \partial^{\nu} T}{1+(\partial T)^{2}}\right) \partial_{\mu} \partial_{\nu} T=-\frac{V^{\prime}}{V}\left(1+(\partial T)^{2}\right) .
$$

From (53) we deduce that contrary to popular prejudice: the tachyon is not a tachyon! If we define the tachyon co-metric by

$$
\left(G^{-1}\right)^{\mu \nu}=g^{\mu \nu}-\frac{\partial^{\mu} T \partial^{\nu} T}{1+(\partial T)^{2}},
$$

so that

$$
\left(G^{-1}\right)^{\mu \nu} G_{\nu \lambda}=\delta_{\lambda}^{\mu},
$$

we see that the characteristic cones of (53) are given by the co-metric $\left(G^{-1}\right)^{\mu \nu}$ and the rays by the metric $G_{\mu \nu}$. It is these which govern the speed of propagation of small disturbance around some background, as may readily be seen by linearising (53). According to the general theory of hyperbolic partial differential equations, the characteristic cones also govern the maximum speed of any possible signal. Note that the characteristic cones do not depend in any way upon the potential $V(T)$, but just on the coefficients of the highest derivative term in the equations of motion. Thus for example linearizing around flat spacetime, we see that the maximum signal speed is that of light because $g_{\mu \nu}$ and $G_{\mu \nu}$ coincide in that case. However in general they will not coincide with the standard Einstein cone $g_{\mu \nu}$ and co-cone $g^{\mu \nu}$, in other words, in a non-trivial tachyon background, tachyon fluctuations will travel at a different speed from that of light. Nevertheless even in this case, because

$$
G_{\mu \nu} l^{\mu} l^{\nu}=\left(l^{\mu} \partial_{\mu} T\right)^{2},
$$

for any vector $l^{\mu}$ lying on the Einstein cone, i,e, such that

$$
g_{\mu \nu} l^{\mu} l^{\nu}=0,
$$


it is clear that the Einstein cone lies outside or on the tachyon cone, which we denote by

$$
g_{\mu \nu} \geq G_{\mu \nu}
$$

and no super-luminal propagation is ever possible.

The energy momentum tensor $T^{\mu \nu}$ of the tachyon takes the form:

$$
T^{\mu \nu}=-V \sqrt{1+(\partial T)^{2}}\left(G^{-1}\right)^{\mu \nu}
$$

from which one deduces from (58) that it satisfies the dominant energy condition, with respect to the Einstein metric $g_{\mu \nu}$ (as well in fact with respect to the metric $G_{\mu \nu}$ ). which according to a result of Hawking, ensures that if the tachyon vanishes at time zero outside some compact set, then it vanishes outside the future of that compact set. Specifically, in a local frame in which $T$ depends only upon time,

$$
\rho=\frac{V(T)}{\sqrt{1-\dot{T}^{2}}} \quad P=-V(T) \sqrt{1-\dot{T}^{2}} .
$$

Thus

$$
P=-\frac{V^{2}(T)}{\rho} .
$$

Note that because of the square root, $|\dot{T}|$ can never exceed unity. If it were the case that $V(T)$ were a constant, independent of $T$ then (61) is the equation of state of what is called a Chaplygin gas. As a pedagogic warm up for the real thing, it is interesting to review the cosmology of such a gas as worked out by Karmenshik, Moshella and Pasquier [29]. It is a special case of what have been called "k-essence" cosmologies 31.

\section{Chaplygin Cosmology}

We set $P=-\frac{A}{\rho}, \quad A>0$. The first law (15) may be integrated to give the density $\rho$ as a function of the scale factor $a$. One gets

$$
\rho=\sqrt{A+\frac{B}{a^{6}}}
$$

where $B$ is an integration constant which we take to be positive. Clearly, at large scale factor, i.e at late times, we have a cosmological term

$$
\rho=-P=\text { constant }
$$

At small scale factor we have a dust-like behaviour, $P=0$, and

$$
\rho \propto \frac{1}{a^{3}} .
$$


The scale factor can thus make a smooth transition from a matter dominated form $a \propto t^{\frac{2}{3}}$ to an exponentially inflating form $a \propto \exp H_{\infty} t$ with $H_{\infty}=$ $\sqrt{\frac{8 \pi G \sqrt{A}}{3}}$.

At large scale factor we have corrections to the pure cosmological term:

$$
\begin{aligned}
\rho & \approx \sqrt{A}+\sqrt{\frac{B}{4 A}} \frac{1}{a^{6}}, \\
P & \approx-\sqrt{A}+\sqrt{\frac{B}{4 A}} \frac{1}{a^{6}} .
\end{aligned}
$$

Thus at late times there is a small admixture of stiff matter.

\section{Tachyons and the strong energy condition}

It is clear that at high density, both the Chaplygin and the tachyon case violate the strong energy condition. In fact

$$
\rho+3 P=\frac{V}{\sqrt{1-\dot{T}^{2}}}\left(3 \dot{T}^{2}-2\right) .
$$

Thus the strong energy condition fails if $|\dot{T}|<\sqrt{\frac{2}{3}}$.

\section{Coupling to gravity}

We assume that the relevant action is

$$
\int d^{4} x\left(\frac{R}{16 \pi G} \sqrt{-\operatorname{det} g_{\mu \nu}}-V \sqrt{-\operatorname{det} G_{\mu \nu}}\right) \quad \text { plus boundary term. }
$$

Substituting in the Raychaudhuri and Friedman equations gives

$$
\begin{gathered}
\frac{\ddot{a}}{a}=\frac{8 \pi G}{3} \frac{V(T)}{\sqrt{1-\dot{T}^{2}}}\left(1-\frac{3}{2} \dot{T}^{2}\right), \\
\frac{\dot{a}^{2}}{a^{2}}+\frac{k}{a^{2}}=\frac{8 \pi G}{3} \frac{V}{\sqrt{1-\dot{T}^{2}}} .
\end{gathered}
$$

The equation of motion for the tachyon field is

$$
\frac{\ddot{T}}{1-\dot{T}^{2}}+3 \frac{\dot{a}}{a} \dot{T}+\frac{V^{\prime}}{V}=0
$$

The first law becomes

$$
\dot{\rho}+\frac{3 \dot{a}}{a} \dot{T}^{2} \rho=0
$$


Since $|\dot{T}| \leq 1$ we deduce from (72). that

$$
\left.(\rho \dot{a})^{3}\right) \leq 0
$$

Now let us suppose that we set $T$ off some where near the top of the potential at positive $T$ and $0<\dot{T}<\sqrt{\frac{2}{3}}$. Let also suppose that $k=0$ and that $V(T)$ takes its minimum value of zero at infinity. The tachyon field will increase monotonically, eventually reaching infinity with $\dot{T}=1$. Initially the scale factor will accelerate but this acceleration is self-limiting, eventually become negative ( once $\dot{T}>\sqrt{\frac{2}{3}}$ ) and slowing down like pressure free matter. From (73) we deduce that the pressure falls dramatically (at least exponentially for reasonable choices of $V(T))$.

\section{Shortcomings}

Many authors have pointed out the shortcomings of the rolling of the tachyon as a mechanism for inflation [33. They include

- The natural scale of the model is the Planck or String scale. Planck scale inflation will in general give fluctuations which are too large.

- If the mass scale near the local maximum at the origin is too large, then insufficient inflation will result before the tachyon field nears the minimum value of the potential.

- The model requires fine tuning so as to avoid being becoming matter dominated before there is time for a hot radiation era during which nucleosynthesis takes place. Alternatively, if one wants the tachyon to act as the cold dark matter apparently seen at the present time, again one seems to require an element of fine-tuning.

- Because the tachyon does not oscillate in a potential well at the end of rolling, the usual mechanism for reheating does not operate.

Although these are all perfectly valid objections, in my opinion, they ignore

- The provisional and approximate nature of the model.

- The incomplete nature of the modelling of the relation between the open and closed string sectors.

For these reasons, I feel that it is too early to abandon completely the idea that the tachyon may have a role to play in cosmology, particularly at very early times. However, as we shall see, if those times were of Planck scale, there remain difficulties in accommodating any such model with the absence of large gravitational wave perturbations. Before passing to that, I wish to make some pedagogic comments on the issue of fine tuning. 


\section{Fine tuning, initial conditions and Anthropic Considerations}

Issues of fine tuning in cosmology, as opposed to particle physics, are concerned with plausible initial conditions in real time rather than the evolution of the renormalization group equations with energy scale. At the classical level, this requires some sort of a priori probability distribution on the space of Cauchy data. At the quantum level, some sort of a priori measure on the space of initial quantum states, that is an a priori density matrix against which to test the plausibility of various "proposals" for the wave function of the universe [46]. To make this quantitative, rather than just a description of personal prejudice, seems to be extremely difficult.

Even at the classical level, and for a finite number of degrees of freedom, such as we have in the model discussed above, this is an extremely tricky business. We have in effect a "mini-superspace" model, with the structure of a Hamiltonian system with a constraint. The constraint is that the flow $(q(t), p(t))$ in a $2 n$ dimensional phase space $\mathcal{P}$ is confined to the $(2 n-1)$ - dimensional submanifold $\Gamma$ on which the Hamiltonian vanishes

$$
\left.H(p, q)\right|_{\Gamma}=0 .
$$

Precisely because we have an autonomous dynamical system on $\Gamma$ we know that given any present condition, there must be some initial conditions which gave rise to it, namely any point on the trajectory or classical history through our present point. Thus we cannot expect a complete "chaotic" style explanation of our present condition: that all initial conditions will inevitably give rise to it. This would only be mathematically possible if we consider what happens if the time went strictly to infinity, but clearly this is not true in cosmology. Rather we need a purely classical measure on the possible histories. This has been provided to us by Liouville and was introduced for this purpose in 22] (see [43] for its independent introduction and use in the context of Bianch-IX models). We first seek to capture all the trajectories on $\Gamma$ by cutting with a $2 n-2$ dimensional surface $S$ intersecting each trajectory once and only once. $S$ is the space of classical histories and the point is that the Liouville or symplectic measure $d^{n} p d^{n} q$ on $\mathcal{P}$ descends to $S$ via a construction called the Symplectic or Marsden-Weinstein quotient.

Using this symplectic measure one may now break $S$ into two domains, one, $S_{\text {yes }}$, with histories close enough to what we believe our own to have been and, those $S_{\text {no }}$ unlike our own.

We can now evaluate the measure and hence probabilities, according to Liouville. The problem is that typically both

$$
\operatorname{measure}\left(S_{\text {yes }}\right)=\infty
$$

and

$$
\operatorname{measure}\left(S_{\text {no }}\right)=\infty \operatorname{.~}
$$


In other words quantities like for example the "probability of sufficient inflation" which are constructed form ratios of these infinite quantities are just not well defined 44, 45.

This problem, which arises even in the simplest finite dimensional case, and is essentially an infra-red problem having nothing to do with the usual need to impose an ultra-violet cutoff in quantum theory, gets much much worse if one goes to the full field theory with its infinite number of degrees of freedom. Similarly, in the quantum theory, even of one particle, one arrives at the problem that one cannot easily normalise density matrices, and certainly not the unit density matrix, which represents complete ignorance. It is for these reasons I am extremely skeptical of claims that the Anthropic Principle can ever be elevated to the level of a precise quantitative tool. On the other hand, the Anthropic Principle does seem to give a very convincing explantion of why the world is $3+1$-dimensional. Interesting renormalizable quantum field theories of the sort we use to describe intelligent life cease to exist in higher dimensions. This comment is particularly relevant to brane scenarios in which our universe may contain branes of different dimensions. All may have excitations propagating on them described by quantum field theories but only on 3-branes will Nobel prizes be awarded for their discovery [21].

\section{Tachyon Condensation}

The most optimistic scenario is that

- All open string states get confined in the true tachyon ground state.

- Closed string states arise as flux tubes or some sort of related solitonic or non-perturbative excitations.

To implement this idea is the ambition of many people. It is a major challenge in string theory. In what follows, I will offer a commentary on how this might look like at the level of an effective classical field theory. More details of the underlying microphysics may be found in for instance [36] 24].

Since the precise tachyon Lagrangian is not completely known at present, although there exist a number of impressive calculations, and in any case any

actual Lagrangian may change under field redefinitions, we consider a general one

$$
L=L(T, y), \quad y=g^{\mu \nu} \partial_{\mu} T \partial_{\nu} T .
$$

From a cosmological point of view such Lagrangians correspond to what is called "k-essence", a pun on "quintessence" 31].

\section{Carollian Confinement}

The confinement of open string states may be given a rather elegant kinematic description in terms of an old, but hitherto unused, idea. 
The Poincaré group $E(n-1,1),{ }^{1}$ has two important Inönü-Wigner contractions which occur in the limiting cases when $c \uparrow \infty$ or $\downarrow 0$. The former case corresponds to the Galilei group when we have instantaneous propagation and action at a distance with fields satisfying elliptic partial differential equations, the latter, which is less well known, is called the Carroll group [1, 2] and corresponds to the case of no propagation at all. Fields at each spatial point evolve independently and are typically governed by ordinary differential equations with respect to just the time variable. For that reason, this case often arises as the symmetry group of an approximation scheme in which spatial derivatives are ignored compared with time derivatives. Such approximation schemes are sometimes called "velocity dominated".

Geometrically the Galilei group arises when the future light cone flattens out to become a spacelike hyperplane. The Carroll group arises when it collapses down to a timelike half line. In the Galilean case only the contravariant metric tensor has a well defined limit as $c \uparrow \infty$ :

$$
\eta^{\mu \nu} \rightarrow \operatorname{diag}(0,1,1, \ldots, 1)
$$

and the limiting spacetime structure is called a Newton-Cartan spacetime. In the case of the Carollian limit it is the covariant metric tensor which survives

$$
\eta_{\mu \nu} \rightarrow \operatorname{diag}(0,1,1, \ldots, 1)
$$

and one has a Carrollian spacetime.

Now let's look at Sen's tachyon metric in the limit of tachyon condensation, i.e. in the limit that $|\dot{T}| \rightarrow 1$. We have

$$
\begin{aligned}
G_{\mu \nu} & =g_{\mu \nu}+\partial_{\mu} T \partial_{\nu} T \\
& =\operatorname{diag}\left(-1+\dot{T}^{2}, 1,1,1\right) \\
& \rightarrow \operatorname{diag}(0,1,1,1)
\end{aligned}
$$

Clearly as $|\dot{T}| \rightarrow 1$, the cone defined by $G_{\mu \nu}$ squeezes onto a half-line and no open tachyonic excitations can propagate. If one thinks of these as sound waves, then the speed of sound goes to zero and hence the pressure drops to zero.

In fact, one may consider a more general tachyon Lagrangian, possibly coupled to a Born-Infeld vector field $A_{\mu}$ with field strength $F_{\mu \nu}=\partial_{\mu} A_{\nu}-\partial_{\nu} A_{\mu}$.

$$
L=-V(T) \sqrt{-\operatorname{det}\left(g_{\mu \nu}+F_{\mu \nu}\right)} \mathcal{F}(z),
$$

with

$$
z=\left(G_{\text {open }}^{-1}\right)^{\mu \nu} \partial_{\mu} T \partial_{\nu} T
$$

where the open string co-metric is given by

$$
\left(G_{\text {open }}^{-1}\right)^{\mu \nu}=\left(\frac{1}{g+F}\right)^{(\mu \nu)} .
$$

\footnotetext{
${ }^{1}$ sometimes referred to as the inhomogeneous Lorentz group and such written as $I S O(n-$ $1,1)$
} 
We know that in general, the open string metric $\left(G_{\text {open }}\right)_{\mu \nu}$ lies inside or on the closed string metric $g_{\mu \nu}$, coinciding along two special null directions $l^{\mu}$ such that $F_{\mu \nu} l^{\nu}=0$. It also seems to satisfy a version of the Equivalence Principle, it is universal for all open string states, just as the closed string metric satisfies the standard Einstein Equivalence Principle: it is universal for all closed string states.

For the time being lets set $F_{\mu \nu}=0$. We then have in Sen's case

$$
L=V(T) \sqrt{1+y},
$$

or from boundary string conformal field theory (BSFT)

$$
L=e^{-\frac{1}{2} T^{2}} \mathcal{F}(y)
$$

with

$$
\mathcal{F}(y)=\frac{y 4^{y} \Gamma(y)^{2}}{2 \Gamma(2 y)} .
$$

In general the energy momentum tensor is

$$
T^{\mu \nu}=L g^{\mu \nu}-2 L_{y} \partial^{\mu} T \partial^{\nu} T
$$

thus

$$
\rho=2 y L_{y}-L, \quad P=L .
$$

The dominant energy condition will hold as long as

$$
2 y L_{y}-L \geq 0 .
$$

The equations of motion are

$$
\left(G^{-1}\right)^{\mu \nu} \partial_{\mu} \partial_{\nu} T=\frac{L_{T}}{2 L_{y}},
$$

and the propagation co-metric given by

$$
\left(G^{-1}\right)^{\mu \nu}=g^{\mu \nu}+\frac{2 L_{y y}}{L_{y}} \partial^{\mu} T \partial^{\nu} T
$$

and the propagation metric given by

$$
G_{\mu \nu}=g_{\mu \nu}-\frac{2 L_{y y}}{L_{y}+2 L_{y y}} .
$$

Thus we shall get causal propagation if

$$
\frac{2 L_{y y}}{L_{y}} \leq 0 .
$$

Using these formulae one may check that not only Sen's energy momentum tensor but also that coming from BSFT satisfy both the strong energy condition 
and have causal propagation. More importantly for the present considerations, one may check that as we approach the condensate, $y \rightarrow-1$, the metric becomes Carollian. In general we have

$$
G_{\mu \nu}=\operatorname{diag}\left(-1-\dot{T}^{2} \frac{2 L y y}{L+y+2 L_{y} y}, 1,1,1\right) .
$$

It follows that as long as $\left|L_{y y}\right| \rightarrow \infty$ as $y \rightarrow-1$ then

$$
G_{\mu \nu}=\operatorname{diag}(0,1,1,1) \text {. }
$$

In fact in the case of BSFT,

$$
L \rightarrow-\frac{1}{2} \frac{1}{(1+y)} .
$$

It is an attractive extrapolation from this example to speculate that, although the tachyon metric may not enjoy the same universality properties as the open string metric [5, 12, 6, 7, the Carollian confinement property described here is universal.

\section{Inclusion of Fluxes}

What happens if we consider a case in which $F_{\mu \nu} \neq 0$ ? One way is to generalise Sen's action to

$$
L=V(T) \sqrt{-\operatorname{det}\left(g_{\mu \nu}+F_{\mu \nu}+\partial_{\mu} T \partial_{\nu} T\right)}
$$

In this case the tachyon field is on the same footing as a transverse scalar in the Dirac-Born-Infeld action for a brane. Alternatively one could use the full BSFT action given above. In both cases we look for a solution with a constant electric field and find that the condensed state at $V(T) \rightarrow 0$ is now given by

$$
\dot{T}^{2}+E^{2}=1
$$

with $E=|\mathbf{E}|$. To understand the dynamics it is, as in a previously studied case, convenient to pass to the Hamiltonian formulation of the theory. Define the conjugate variables

$$
\mathbf{D}=\frac{\partial L}{\partial \mathbf{E}}, \quad P=\frac{\partial L}{\partial \dot{T}} .
$$

The Hamiltonian density is

$$
\mathcal{H}=\mathbf{D} \cdot \mathbf{E}+P \dot{T}-L .
$$

In the Sen case one discovers that

$$
\mathcal{H}=\sqrt{\mathbf{D}^{2}+P^{2}+(\mathbf{D} . \nabla T)^{2}+\left(F_{i j} D_{j}+\partial_{i} T\right)^{2}+V^{2} \operatorname{det}\left(\delta_{i j}+F_{i j}+\partial_{i} T \partial_{j} T\right)}
$$

Obviously there is a smooth $V \downarrow 0$ limit.

One may now investigate the propagation of small fluctuations in the limit when $\dot{T}^{2}+E^{2}=1$. One finds that 
- Propagation in directions orthogonal to $\mathbf{E}$ is suppressed.

- Propagation along the direction of parallel to $\mathbf{E}$ has speed $\pm E$. This is just what one expects of a fluid of parallel flux tubes or strings and is consistent with earlier work on string fluids

We recover that system by dropping the tachyon. One gets, in the limit $V \downarrow 0$

$$
\mathcal{H}=\sqrt{\mathbf{D}^{2}+(\mathbf{D} \times \mathbf{B})^{2}} .
$$

One should note that the energy of a static electric flux line with $\mathbf{B}=0$ is proportional to its length, as expected, because in that case $\mathcal{H}=|\mathbf{D}|$. Now,

$$
\mathbf{H}=\frac{\mathbf{B D}^{2}-\mathbf{D}(\mathbf{B} \cdot \mathbf{D})}{\sqrt{\mathbf{D}^{2}+(\mathbf{D} \times \mathbf{B})^{2}}}
$$

and

$$
\mathbf{E}=\frac{\mathbf{D}+\mathbf{D B}^{2}-\mathbf{B}(\mathbf{B} \cdot \mathbf{D}}{\sqrt{\mathbf{D}^{2}+(\mathbf{D} \times \mathbf{B})^{2}}}
$$

It follows that

$$
\text { D.H }=0, \quad \mathbf{D}^{2}-\mathbf{H}^{2}>0 .
$$

Thus if one constructs an Ampère tensor $K=\frac{1}{2} K_{\mu \nu} d x^{\mu} \wedge d x^{\nu}$ from $(\mathbf{D}, \mathbf{H})$ in the same way that one construct the Faraday tensor $F_{\mu \nu}$ from $(\mathbf{E}, \mathbf{B})$ one gets.

$$
\operatorname{det} K_{\mu \nu}=0, \Leftrightarrow K \wedge K=0,
$$

and

$$
K_{\mu \nu} K^{\mu \nu}<0 .
$$

Thus the two-form $K$ is simple and defines a distribution of timelike 2-planes in the tangent space at each point of spacetime. The field equations

$$
d K=0,
$$

imply that this distribution is integrable, i.e. that spacetime is foliated by timelike 2-surfaces tangent to $K$. Physically one may identify these surfaces with the world sheets of a fluid of electric flux tubes. Their energy momentum tensor is given by

$$
T^{\mu \nu}=-\frac{K^{\mu}{ }_{\lambda} K^{\nu \lambda}}{\sqrt{-\frac{1}{2} K_{\alpha \beta} K^{\alpha \beta}}}
$$

For a static flux tube

$$
T_{\mu \nu}=\left(\begin{array}{cccc}
\mathcal{H} & 0 & 0 & 0 \\
0 & -\mathcal{H} & 0 & 0 \\
0 & 0 & 0 & 0 \\
0 & 0 & 0 & 0
\end{array}\right)
$$


This has a tension equal to the energy density in the direction of the electric field and zero pressure transverse to the electric field, again as expected.

Although this classical model exhibits a string fluid behaviour with flux tube solutions, there remain some obvious problems. Neither the thickness nor the value of the flux is determined. Hopefully, this will emerge in some future quantum mechanical treatment.

\section{Carroll versus Galilei}

It is clear that there is some sort of duality between the Carroll and Galilei groups. The purpose of this penultimate section is to describe this duality in a geometrical way by lifting there action up to one higher spatial dimension and exhibiting both groups as subgroups of the Poincaré group $E(n, 1)$. By passing to one further temporal dimension one may embed the Poincaré group in the Conformal group $E(n, 2)$. The description of the Carroll group I am about to describe is not new, in that it is very briefly described in [38, which is largely about the lifting of the action of the Galilei group to one higher dimension first given by Kunzle and Duval (see 38] for original references). The reason for returning to the subject here is the hope that it may afford some more insight into the properties of the tachyon condensate.

In fact, as we shall see from our discussion, the Carroll group will also emerge naturally in brane-dymanics in the limit that the brane world volume becomes lightlike. Another direct consequence of our analysis, is that one sees the Carroll group emerging naturally in the isometry group of certain pp-wave spacetimes which have been intensively studied of late. Much earlier the possible role of Carrollian spacetimes near spacetime singularities and in the so called strong coupling (i.e. large inverse gravitational tension $\frac{G}{c^{4}}[26]$ ) limit of General Relativity has been discussed by Henneaux [16, 17] and this is closely related to recent work on Kac-Moody symmetries in M-theory [28]. Finally one might hope that just as the Galilei-covariant theories can easily be constructed using the null reduction described below, so one might hope to construct Carrollcovariant theories using the dual related ideas. This might lead to an extension of the work in $[\underline{34}]^{2}$.

\subsection{The Lift}

Let us start with the well studied Galilei case. The basic idea is to start with flat Minkowski spacetime $\mathbb{E}^{n, 1}$ whose metric written in double null coordinates $\left(u, v, x^{i}\right), i=1,2, \ldots, n-1$, is

$$
d s^{2}=-2 d u d v+d x^{i} d x^{i} .
$$

The Lie algebra of the Poincaré group $\mathfrak{e}(n, 1)$ is spanned by the Killing vector fields generating the Lie algebra of the Euclidean group $\mathfrak{e}(n-1)$, translations

${ }^{2}$ I am grateful to Joaquim Gomis for drawing my attention to the possible interest of Carrollian string theories 
and rotations

$$
P_{i}=\partial_{i} \quad L_{i j}=x_{i} \partial_{j}-x_{j} \partial_{i},
$$

two null translations and one boost

$$
U=\partial_{u}, \quad V=\partial_{v} \quad N=u \partial_{u}-v \partial_{v},
$$

and two further sets of boosts

$$
U_{i}=u \partial_{i}+x_{i} \partial_{v} \quad V_{i}=v \partial_{i}+x_{i} \partial_{u} .
$$

There is an obvious symmetry under inter-changing $u$ and $v$ induced by reflection in the timelike $(n-1)$ - plane $u=v$.

To obtain the Bargmann group, the central extension of the Galilei group, we ask for the subgroup which commutes with the null translation generated by $V=\partial_{v}$. This is generated by $\left\{P_{i}, L_{i j}, U, V, U_{i},\right\}$. The Galilei group is obtained by taking the quotient by the null translation group $\mathbb{R}$ generated by $V$. It is easy to see that the Galilei group acts on the quotient $\mathbb{E}^{n, 1} / \mathbb{R}$ which may be identified with a Newton-Cartan spacetime $M^{n}$, the coordinate $u$ playing the role of Newtonian absolute time. The generators $V_{i}$ are Galilean boosts. Because

$$
\left[P_{i}, U_{j}\right]=\delta_{i j} V
$$

they commute with spatial translations (modulo $V$ ) but not with time translations

$$
\left[U, U_{i}\right]=P_{i}
$$

We may think of this construction in terms of a Kaluza-Klein type reduction in which we think of $\mathbb{E}^{n, 1}$ as a fibre bundle with projection map

$$
\pi: \mathbb{E}^{n, 1} \rightarrow M^{n}
$$

given by $\left(u, v, x^{i}\right) \rightarrow\left(u, x^{i}\right)$. However in contrast to the usual case, the fibres are lightlike. Using the map $\pi$ one may push forward the Minkowski co-metric on $\mathbb{E}^{n-1}$ down to the Newton-Cartan spacetime to give the degenerate co-metric (78).

To obtain the Carroll group, we ask instead for the subgroup of the Poincaré group which leaves invariant the null hyperplane $u=$ constant. This is generated by $\left\{P_{i}, L_{i j}, V, U_{i}\right\}$. Now the null coordinate $v$ plays the role of time. The Carollian boosts $U_{i}$ commute with time translation

$$
\left[V, U_{i}\right]=0
$$

but by (113) they no longer commute with spatial translations $P_{i}$. In fact one obtains a Heisenberg sub-algebra with the time translations being central. From an algebraic point of view the Carrol and Galilei groups differ only in the choice of generator of time translations: one picks either $V$ or $U$.

One may think of the null hyperplane $u=$ constant as the image under the embedding map

$$
x: M^{n} \rightarrow \mathbb{E}^{n, 1},
$$


such that $\left(v, x^{i}\right) \rightarrow$ (constant, $\left.v, x^{i}\right)$, of a Carollian spacetime time. The pull back of the Minkowski metric gives the degenerate Carrollian metric (79). Thus the duality relating the cases is between an immersion $x$ (117) and a submersion $\pi$ (115) and interchanges domain and range.

\subsection{Plane Waves}

One may generalize the idea of a Newton-Cartan spacetime to include gravitational fields by replacing $\mathbb{E}^{n, 1}$ in the construction above by an $n+1$ dimensional spacetime admitting a covariantly constant null Killing field. In the context of Galilei kinematics such spacetime is said to admit a Bargmann structure. Physically it corresponds to a plane-fronted gravitational wave with parallel rays, or pp-wave for short. In harmonic coordinates, the metric takes the form

$$
d s^{2}=-2 d u d \tilde{v}+H(\tilde{x}, u) d u^{2}+d \tilde{x}^{i} d \tilde{x}^{i} .
$$

In the special case that the function $H(\tilde{x}, u)$ is quadratic in $\tilde{x}$, the isometry group is enhanced from $\mathbb{R}$ to a $(2 n+1)$-dimensional Heisenberg group. This acts on null hypersurfaces and is a subgroup of the Carroll group. Gravitational waves of this type are called plane waves and as emphasised by Bondi, Pirani and Robinson in the case $n=3$ the symmetry group and number of polarisation states coincide exactly with what one obtains from linearised theory and also for plane electromagnetic waves. If $n>3$ the symmetry groups also coincide but the number of polarisation states of course differ.

\section{Concluding Remarks}

Given the speculative picture of the tachyon condensate outlined above, it is clear that issues such as "reheating " and gravitational wave production may be very different from what they are in the standard picture. It therefore seems to me to be premature to rule out a rôle for the tachyon in cosmology.

Consider for instance, a world which initially contains both open string states and hence necessarily closed string states. One could envision an initial Open String Era during which the system rolled down to the true vacuum, a tachyon condensate in which all open string states suffer Carrollian Confinement, there propagation cone collapses onto a half-line and thereafter only closed string states can propagate. We now enter the Closed String Era in which presumably the open strings eventually reassemble themselves to give the standard model during some Primordial Radiation Era. After this point a more or less conventional inflationary scenario could have set in.

One might think that we might never have access to any information form before the confining phase transition separating the Open String Era from the Closed String Era. However this may not be completely correct. As I described earlier, we can treat in a rough way the cosmological rolling to wards the tachyon condensate using a simple FLRW model. We can also consider gravitational wave perturbations around that background configuration. If these gravitational 
waves can penetrate beyond the confining phase transition, then they should be observable today and this raises difficulties with the observational data. This is of course just the basic problem with all primordial inflationary models. However in this case things might just be different, because the graviton is just a closed string state and closed strings are supposed to be topological excitations, flux tubes in the true open string vacuum. If that is true then the gravitons we can see today must, in some sense have been created then. More particularly, it is not obvious that any gravitons created earlier could have passed through the Carrollian barrier. If that is true, then we need not worry about the present limits.

It appears that a complete theoretical treatment of a scenario like this is way out of reach of present day techniques in String Theory. Some relevant new ideas involving the Wheeler-De-Witt equation may be found in 37. Thus it

must remain at present a speculation. Nevertheless it seems to me well worth bearing in mind, if only as a challenge of our powers of theoretical analysis.

\section{References}

[1] J. M. Lévy-Leblond, Une nouvelle limite non-relativiste du group de Poincaré, Ann Inst H Poincaré 3 (1965) 1-12

[2] V. D. Sen Gupta, On an Analogue of the Galileo Group, Il Nuovo Cimento 54 (1966) 512-517

[3] H. Bacry and J. M Lévy-Leblond, Possible Kinematics J Math Phys 9 (1967) 1605

[4] H. Bacry and J. Nuyts, Classification of Ten-Dimnsional Kinematical Groups with Space Isotropy J Math Phys 27(1986) 2455

[5] G. W. Gibbons and C.A. R Herdeiro, Born-Infeld Theory and Stringy Cosmology, Phys Rev D63 (2001) 064006 hep-th/0008052

[6] G. W. Gibbons, Aspects of Born-Infeld Theory and String/M-Theory hep-th/0106059

[7] G. W. Gibbons, Pulse Propagation in Born-Infeld theory, the World Volume Euqivalence Principle and the Euqation od State of the Chaplygin Gas, hep-th/0104015

[8] S. W. Hawking, The Conservation of Matter in General Relativity Comm Math Phys 18 (1970) 303-306

[9] B. Carter, Energy Dominance anad the Hawking Ellis vacuum conservation theorem gr-qc/0105010

[10] S. Gao and R. M. Wald, Theorems on gravitational time delay and related issues, Class Quant Grav 17 (2000) 4999-5008 gr-qc/0007021 
[11] D. N. Page, S. Surya and E. Woolgar, Positive Mass from Holographic Causality, hep-th/0204198

[12] G. W. Gibbons and P. C. West, The metric and strong coupling limit of the M5-brane, J Math Phys 42 (2001) 3188-3208 hep-th/0011149

[13] S.W. Hawking Mon Not Roy astr Soc 142 (1969) 129

[14] J.D. Barrow, R. Juszkiewicz and D. H. Sonoda, Universal rotation: how large can it be? Mon Not Roy astr Soc 213 (1985) 917-943

[15] J. Ellis and K. A. Olive, Inflation can solve the rotation problem Nature 303 (1983) 679

[16] M. Henneaux,Bull Soc Math Belg 31 (1979) 47-63

[17] M. Henneaux,Acad. Roy. Belg., Bull. Sci. (5) 68 (1982) 940-971

[18] M Martin, Ultra-fast particles inflated Universe, physicists say Science News Week 8-May (2001)

[19] A. Borde, A. H. Guth and A. Vilenkin, Inflation is not past-eternal, gr-qc/0110012

[20] Class Quantum Gravity 15 volume 9 (1998)

[21] G. W. Gibbons, The Dimensionality of Spacetime, in String Theory Quantum Cosmology and Quantum Gravity Interable and Conformal Invraint Theories, eds N Sanchez and H de Vega ( World Scientific, Singapore) 16-25 (1987)

[22] G. W. Gibbons, S.W. Hawking and J.M Stewart, A Natural Measure on the Set of all Universes Nucl Phys B281 (1987) 736-751

[23] G. W. Gibbons, Cosmological evolution of the rolling tachyon Phys Lett B537 (2002) 1-4 hep-th/0204008

[24] G. W. Gibbons, K Hashimoto and Piljin Yi, Tachyon condesates, Carrollian contractions of the Lorentz group and fundamental strings JHEP (2002) 0209: 061 hep-th/0209034

[25] G. W. Gibbons, K Hori and Piljin Yi, Nucl Phys B596 (2001) 136-150 hep-th/0009061

[26] G. W. Gibbons, The Maximum Tension Principle in General Relativity, Foundations of Physics in press hep-th/021009

[27] A. Linde Phys Lett 162B (1988) 281

[28] T. Damour, M .Henneaux and H. Nicolai, Cosmological Billiards hep-th/0212256 
[29] A. Kamenshchik, U. Moshella and V. Pasquier An alternative to Quintessence gr-qc/0103004 Can the Chaplygin gas be a plausible model for dark energy? astro-ph/02009395; R. Collister et al., Dark Energy, Dark matter and the Chaplygin gas gr-qc/0210079

[30] I. Ozsvath and L. E. Schucking Nature 193(1962) 1168; Ann Phys (NY) 55 (1969) 166

[31] Armendariz-Picon, T. Damour, V. Mukhanov Phys Lett B458 (1999)209 hep-th/9904075

[32] F. J. Tipler, General Relativity, Thermodynamics and the Poincaré Cycle Nature 280 (1979) 203-205; General Relativity and the Eternal Return, in Essays in General Relativity Academic Press (1980) 21-35; The Physics of Immortality, Anchor Books (1995)

[33] A. Linde, L. Kofman and A. Starobinsky, Prospects and Problems of Tachyon Cosmology hep-th/0204187 L. Kofman and A .Linde, Problems with Tachyon Inflation hep-th/0205121 M. Fairbairn and M. H. G. Tytgat Inflation from Tachyon Fluid ? hep-th/0204070 G. Shiu and I. Wasserman, Cosmological Constraints on Tachyon Matter hep-th/0205003 G. Felder, L. Kofman and A. Starobinsky, Caustics in Tachyon matter and Other Born-Infeld Scalars, hep-th/0208019 J Cline, H. Firouzjahl and P. Martineau, Reheating from Tachyon Condensation hep-th/0207156 C. Kim, H. B. Kim and Y. Kim, Rolling Tachyons in String Cosmology hep-th/0210101

[34] J. Gomis and H. Ooguri, Non-relativistic closed String Theory hep-th/0009181

[35] G W Gibbons, Aspects of Supergravity Theories, in Supersymmetry, Supergravity and Related Topics eds. F. del Aguilla, A. Azcarrage and L. E. Ibanez (World Scientific) (1985)

[36] A. Sen, Rolling Tachyon hep-th/ 0203211 Tachyon Matter hep-th/0203255 Field Theory of Tachyon Matter hep-th/0204143

[37] A Sen, Time and the Tachyon hep-th/0209122

[38] C. Duval, G.W. Gibbons, P.Horvathy, Celestial mechanics, conformal structures and gravitational waves Phys Rev D43(1991) 3907-3922

[39] W.-H. Huang, Chronology Protection in Generalized Gödel Spacetime Phys Rev D60 (1999) 06715 hep-th/0209091

[40] G. W. Gibbons, R. Kallosh and A. Linde, Brane World Sum Rules JHEP 0101 (2001) 022 hep-th/0011225

[41] J. L. Synge, Newtonian Gravitational Field Theory Il Nuovo Cimento 8B (1972) 373-390 
[42] M. Levy Nahas, J Math Phys 8(1967) 1211

[43] M Henneaux, Nuovo Cimento Lett 38 (1983) 609

[44] S. W. Hawking and D. N. Page, How probable is inflation? Nucl Phys B298(1988) 789-809

[45] S. Holland and R. M. Wald, Comments on Inflation and Alternative Cosmology hep-th/0210001

[46] G. W. Gibbons and L. P. Grishchuk, What is a typical wave function for the universe? Nucl Phys B313 (1989) 736-748

[47] P. Fré, M. Trgiante and A. Van Proeyen, $\mathcal{N}=1$ supergravity models with stable de-Sitter vacua hep-th/0301024

[48] G. W. Gibbons and C. M. Hull, De-Sitter Space from Wraped Supergravty Solutions hep-th/0111072

[49] D. Z. Freedman, Supergravity with axial-gauge-invariance Phys Rev D15(1977) 1173-1174 